\title{
Solar diameter measurements from eclipses as a solar variability proxy
}

\author{
David W. Dunham ${ }^{1}$, Sabatino Sofia ${ }^{2}$, Konrad Guhl ${ }^{3}$ and David \\ Herald $^{4}$ \\ ${ }^{1}$ International Occultation Timing Association (IOTA), 7913 Kara Ct., Greenbelt, MD \\ 20770-3016, USA \\ email: dunham@starpower.net \\ ${ }^{2}$ Dept. of Astronomy, Yale Univ. (retired), Williamsburg, VA 23185, USA \\ email: sabatino.sofia@yale.edu \\ ${ }^{3}$ IOTA/European Section, Archenhold Obs., Alt-Treptow 1, 12435 Berlin, Germany \\ email: kguhl@astw.de \\ ${ }^{4}$ IOTA, 3 Lupin Place, Murrumbateman, NSW 2582, Australia \\ email: drherald@bigpond.net.au
}

\begin{abstract}
The widths of total solar eclipse paths depends on the diameter of the Sun, so if observations are obtained near both the northern and southern limits of the eclipse path, in principle, the angular diameter of the Sun can be measured. Concerted efforts have been made to obtain contact timings from locations near total solar eclipse path edges since the mid 19th century, and Edmund Halley organized a rather successful first effort in 1715. Members of IOTA have been making increasingly sophisticated observations of the Baily's bead phenomena near central solar eclipse path edges since 1970 .
\end{abstract}

Keywords. eclipses, photosphere, chromosphere, solar-terrestrial relations, fundamental parameters, Moon

\section{Introduction}

Since thermal relaxation times for the Sun are thousands of years, small variations of the Solar intensity are proportional to small variations of the Solar diameter on decadal time scales. In a combination between observations and theory, reliable values of the relation constant $\mathrm{W}$ are known, that allow transformation of historical variations of radius into variations of the solar luminosity. During the past 45 years, members of IOTA have observed 20 annular and total solar eclipses from locations near the path edges. Baily's beads, whose occurrence and duration are considerably prolonged as seen from path edge locations, were first timed visually, mostly using projection techniques. The edge locations have the advantage that most of the beads are defined by the same features in the lunar polar regions that cause the phenomena at each eclipse.

\section{Past Observations and Results}

Most observers of solar eclipses travel to the central line so that before IOTA's efforts that started with the 1970 March eclipse, there are only a few earlier eclipses observed near the path edges where the results were published in enough detail for useful analysis. Since 1980, large numbers of Baily's beads have been timed mainly from analysis of video recordings. Some of these results are noted by Fiala et al. (1994). Since then, Dunham worked with student interns to analyze most of these published past observations, as well 


\begin{tabular}{|c|c|c|c|c|c|c|c|}
\hline Year & Month & Day & Type & \# Obs. & Method & $\Delta R O, "{ }^{\prime \prime}$ & $\sigma, \pm, "$ \\
\hline 1715 & May & 03 & Total & 3 & visual & -0.5 & 0.2 \\
\hline 1878 & July & 29 & Total & 12 & visual & +0.55 & 0.15 \\
\hline 1900 & May & 28 & Total & 8 & visual & +0.12 & 0.09 \\
\hline 1912 & April & 17 & Hybrid & 26 & photoes & -0.29 & 0.22 \\
\hline 1922 & Sept. & 21 & Total & 4 & visual & +0.2 & 0.4 \\
\hline 1925 & Jan. & 24 & Total & 11 & visual & -0.21 & 0.17 \\
\hline 1966 & May & 20 & Hybrid & 9 & photoes & +0.03 & 0.05 \\
\hline 1970 & March & 7 & Total & 4 & visual \& proj. & -0.05 & 0.12 \\
\hline 1972 & July & 10 & Total & 14 & visual \& proj. & -0.02 & 0.61 \\
\hline 1973 & June & 30 & Total & 7 & visual \& proj. & +0.4 & 0.2 \\
\hline 1976 & Oct. & 23 & Total & 14 & projection & -0.38 & 0.09 \\
\hline 1979 & Feb. & 26 & Total & 21 & visual \& proj. & -0.05 & 0.08 \\
\hline 1980 & Feb. & 16 & Total & 36 & projection & -0.18 & 0.05 \\
\hline 1981 & Feb. & 04 & Annular & 52 & projection & -0.21 & 0.05 \\
\hline 1983 & June & 11 & Total & 26 & video \& proj. & -0.10 & 0.08 \\
\hline 1984 & May & 30 & Hybrid & 27 & video & +0.13 & 0.07 \\
\hline 1987 & March & 29 & Hybrid & 11 & photoes & -0.19 & 0.08 \\
\hline 1987 & Sept. & 23 & Annular & 44 & video & +0.10 & 0.05 \\
\hline 1988 & March & 18 & Total & 20 & projection & -0.13 & 0.14 \\
\hline 1991 & July & 11 & Total & 23 & video & 0.00 & 0.06 \\
\hline 1994 & May & 10 & Annular & 75 & video & -0.25 & 0.03 \\
\hline 1995 & Oct. & 24 & Total & 25 & video & +0.11 & 0.06 \\
\hline 1998 & Feb. & 26 & Total & 44 & video & +0.16 & 0.04 \\
\hline 1999 & Aug. & 11 & Total & 19 & video & -0.03 & 0.04 \\
\hline 2002 & Dec. & 04 & Total & 13 & video & -0.22 & 0.06 \\
\hline 2005 & Oct. & 03 & Annular & 69 & video & -0.06 & 0.03 \\
\hline 2006 & March & 29 & Total & 105 & video & +0.09 & 0.16 \\
\hline
\end{tabular}

Figure 1. Solar radius determinations from solar eclipses. The radius correction, delta-R, is relative to the standard value at 1 A.U., 959.63 arc seconds.

as a backlog of video observations made by IOTA observers. A table of results, analyzed with lunar limb data from "The Marginal Zone of the Moon" Watts (1963), is shown in Figure 1. More detailed results from recent eclipses, with references to earlier work, are given by Sigismondi et al. (2009).

\section{Accuracy of the Solar Radius Corrections}

One-sigma accuracies are given in the last column of the table of Figure 1, but these are only based on the scatter of the observations (usually made by similar techniques) for each eclipse. Some of the best-observed modern eclipses can be used to assess the real accuracy of the results, which are limited mainly by the intensity drop at the Sun's edge, and the consequent uncertainty in defining the edge. A good example was the total eclipse of 1998 February 26 that was video recorded with accurate GPS time insertion by four observers, two near each limit, in Venezuela and Curacao. This resulted in 4 different radius correction (DR) determinations for each combination of pairs of observers, as shown in the table in Figure 2. The top line of Figure 2 is the result for all four observers. \# gives the number of Baily's bead timings for each observer used in the solution, with only beads occurring at high latitude (within 30 degrees of the lunar north and south 


\begin{tabular}{|c|c|c|c|c|c|c|c|cc|}
\hline N1 & $\#$ & N2 & $\#$ & S1 & $\#$ & $\mathbf{S} 2$ & $\#$ & DR & ERROR \\
\hline DD & 15 & R & 18 & RN & 11 & $W W$ & 04 & 0.16 & 0.0542 \\
\hline DD & 15 & - & - & RN & 11 & - & - & 0.26 & 0.0360 \\
\hline- & - & RR & 18 & RN & 11 & - & - & 0.20 & 0.0349 \\
\hline DD & 15 & - & - & - & - & $W W$ & 04 & 0.00 & 0.0494 \\
\hline- & - & PR & 18 & - & - & WW & 04 & -0.07 & 0.0418 \\
\hline
\end{tabular}

Figure 2. Comparison of solar radius corrections (DR) from video recordings of Baily's beads made by different observers at the eclipse of 1998 February 26. Observers were DD = David Dunham and Patricia Rosenzweig near the northern limit, and RN = Richard Nugent and WW = Wayne Warren, Jr. at the southern limit.

poles) considered. ERROR is the standard error for the determination of DR from all of the observations on the line. The other lines give the result for different pairs of observers. Although the standard errors are all only a few hundredths of an arc second for each pair, the consistency of the DR values has a wider spread, 0.33 arc seconds, certainly due to differences in the determination of the "zero level" where a bead event is considered to have just disappeared or reappeared, with the different telescopes, filtration, and cameras used by the observers. We conclude that the real error of the observations is of the order of + and -0.2 arc seconds, over 4 times larger than the formal errors of individual pairs of observations. In addition, direct visual contact timings made near the path edges during earlier eclipses have been found in the literature, and analyzed. A similar consistency check of visual contact timings (reported durations of totality) made by 5 different observers near the limits of the 1878 July eclipse showed an error of + and - 0.4 arc second. Although the observations seem to show small variations from eclipse to eclipse, they are only a little larger than the assessed accuracies. More information is given in the poster presentation associated with this paper; it is available by clicking on the "Solar Diameter Measurement" link on IOTA's 2015 meeting presentations Web page at http://occultations .org/community/meetingsconferences/na/na2015/2015annual-meeting-presentations/.

\section{Future Work}

The results can be improved with a consistent re-analysis of the observations using the much more accurate lunar profile data that are now available from the Japanese Kaguya and NASA's LRO lunar orbiter observations. It seems now that the black-and-white video recordings of Baily's beads that we have used during the past several years can not give good results due to the difficulty in defining the solar edge, as shown in Figure 2. Perhaps less-filtered color recordings will be better, as the red color of the chromosphere becomes evident when the bright photosphere is covered. In a similar way, some astronomers have recorded the flash spectrum, defining the edge when absorption lines turn to emission lines (or measure the inflection point of the intensity light curve of a part of the spectrum not affected by spectral lines), especially by Japanese astronomers at eclipses in 1970 and 1973, and by French astronomers in 2010. IOTA has plans to observe future eclipses, especially the one across the USA in August 2017, with a variety of techniques that were used in the past, to better assess the accuracies of the different observational methods that have been used, and determine systematic differences between them. A separate poster, DEp1.08, gives some information about IOTA's plans for the August 2017 total solar eclipse and can be seen by clicking on the "Plans for 2017 Solar Eclipse" link on IOTA's 2015 meeting presentations Web page. 


\section{Acknowledgements}

Most of the results presented here were from analyses performed at the Johns Hopkins University/Applied Physics Laboratory by student interns from 2004 to 2008, mainly funded by NASA grant NNG04GM60G. We also acknowledge the contributions of many dozens of volunteer observers who often travelled great distances to make the observations, usually at their own expense.

\section{References}

Fiala, A. D., Dunham, D. W., \& Sofia, S. 1994, Solar Phys., 152, 97

Sigismondi, C., Dunham, D. W., Guhl, K., Andersson, S., Bode, H., Canales, O., Colona, P., Farago, O., Fernandez-Ocanya, M., Haupt, M., Herold, C., Nugent, R., Oliva, P., Perello, C., Rothe, W., Rovira, J., Schaefer, T., Schnabel, C., Schwartz, D., Selva, A., Strickling, W., Tegtmeier, A., Tegtmeier, C., Thome, B., \& Warren, W. H. 2009, Solar Phys., 258, 191

Watts, C. B. 1963, Astron. Papers Amer. Ephem., (Washington, DC: US GPO) 17, 1 